\title{
Simulation of Spatial Distributions of Atmospheric Heavy Metals in Xinzhuang and Assessment of Their Health Risks
}

\author{
Yanjiao Chen ${ }^{1}$,Yaobin $\mathrm{Meng}^{2}$, Hongbin Cao ${ }^{1}$ \\ ${ }^{1}$ College of Resources Science \& Technology, Beijing Normal University, Beijing 100875, China \\ 2Academy of Disaster Reduction and Emergency Management, Beijing Normal University, Beijing 100875, \\ China
}

辛庄镇大气重金属浓度分布模拟及健康风险评价

\author{
陈艳姣 ${ }^{1}$, 孟耀斌 ${ }^{2}$, 曹红斌 ${ }^{1}$ \\ ${ }^{1}$ 北京师范大学资源学院, 北京 100875 , 中国 \\ 2 北京师范大学减灾与应急管理研究院, 北京 100875 , 中国
}

\begin{abstract}
The exhaust gas generated by waste incineration plant contains heavy metals. They will harm organ function and human health. The CALPUFF model was used to simulate the transport and diffusion of heavy metals of cadmium $(\mathrm{Cd})$, chromium $(\mathrm{Cr})$, copper $(\mathrm{Cu})$ and nickel (Ni) in atmosphere emitted from exhaust gas of waste incineration plant in Xinzhuang Town in 2013. The spatial distribution characteristics of different heavy metals were analyzed in different seasons, and its potential health risks were evaluated. The results showed that: The concentrations of heavy metals in different seasons decreased in the following order: summer $>$ spring $>$ winter $>$ autumn. The spatial distributions of heavy metals were similar. The concentrations of different heavy metals decreased in the following order: $\mathrm{Cu}>\mathrm{Cd}>\mathrm{Cr}>\mathrm{Ni}$. Atmospheric heavy metals from the waste incineration plant in Xinzhuang caused little risk to the human body.
\end{abstract}

Keywords: heavy metals; spatial and temporal distribution; health risk; CALPUFF; MSWI

\section{摘要}

垃圾焚烧产生的废气中含有重金属, 会危害人体器官 功能, 对健康产生影响。本文通过 CALPUFF 模型模 拟 2013 年辛庄镇垃圾焚烧厂排放的废气中重金属镉、
铬、铜、镍在大气中的迁移扩散, 对不同季节不同重 金属的浓度空间分布进行分析, 并评价其健康风险。 结果表明: 不同季节重金属浓度的大小顺序为夏季> 春季>冬季>秋季; 四种重金属浓度空间分布相似, 浓度的大小顺序为铜 $>$ 镉 $>$ 铬 $>$ 镍; 大气重金属对当地 居民没有造成大的健康风险。

关键词: 重金属; 时空分布; 健康风险; CALPUFF; 垃圾焚烧

\section{1. 引言}

中国城镇化进程飞速向前, 但粗放式的经济发展模式 造成了一系列的环境问题。其中, 工业废气及机动车 尾气排放等产生的大气重金属会污染环境, 并随着呼 吸进入人体, 对人体健康产生严重危害。因此, 有必 要研究大气重金属污染物的迁移扩散过程、浓度空间 分布及其健康风险, 为大气重金属污染控制及健康风 险管理提供依据。

众多国内外学者使用EPA推荐的CALPUFF模型 研究污染物的迁移扩散过程, CALPUFF为三维拉格 朗日烟团扩散模型, 能模拟在非定常、非稳态的时空 变化的气象条件下, 污染物输送、转化和清除过程 [1], 广泛应用于大气污染物扩散模拟和环境风险评估等 领域。它能计算次层网格区域的影响(如地形的影响), 和长距离输送的影响(如由于干湿沉降导致的污染物 清除、化学转变和颗粒物浓度对能见度的影响) [2]。 
Risk Analysis and Crisis Response in Big Data Era (RAC-16)

国内外较多地利用CALPUFF模型来分析颗粒物或 $\mathrm{SO}_{2} 、 \mathrm{NO}_{x}$ 等气体的时空分布特征, 并得出区域人群 的暴露风险[3-9]。

CALPUFF模型既可以模拟一个地区现在的大气 污染物的浓度和污染物对土壤、水体的影响, 亦能够 预测未来几年内该地区的大气污染物的浓度, 并进一 步评价大气污染对动植物的生态风险和人体健康风 险, 进而提出解决建议。但应用于城镇尺度大气重金 属排放的迁移扩散模拟 (考虑干湿沉降) 的研究不多。

本论文以江苏省常熟市辛庄镇为研究区, 采用 CALPUFF模型模拟垃圾焚烧厂排放废气中重金属的 迁移扩散过程, 得到辛庄镇大气中重金属的空间分布, 并计算当地居民大气重金属暴露的健康风险。

\section{2. 研究方法和数据来源}

\section{1 研究区概况}

辛庄镇隶属于江苏省常熟市, 北纬 $31^{\circ} 32^{\prime}$, 东经 $120^{\circ} 41^{\prime}$ ，位于市南部。系长江三角洲冲积平原 [10]。 辛庄镇位于亚热带南部湿润季风区, 光照充沛, 雨量 充足, 无霜期长, 气候宜人。辛庄镇域处纬度适中, 年均温 $15.4^{\circ} \mathrm{C}$, 年均日照时长 $2118.6 \mathrm{~h}$, 占应有日照 时数的一半, 年太阳辐射量 $118.029 \mathrm{Kcal} / \mathrm{cm}^{2}$, 年均 降水量达 $1048.30 \mathrm{~mm}$, 年均相对湿度为 $80 \%[11]$ 。

辛庄镇总面积 104.26 平方千米, 户籍人口 75973 人, 常住人口约 10 万人。乡镇企业以纺织服装、机 械装备、生物医药、新能源 (光伏) 产业为主, 但规 模不大。主要的大气重金属污染来源为常熟市最早在 该镇建立的垃圾焚烧厂。该厂位于辛庄镇的东北角, 处理常熟市产生的大部分垃圾。具体位置为图 1 中标 注点源, 垃圾焚烧厂的遥感影像如图 2。

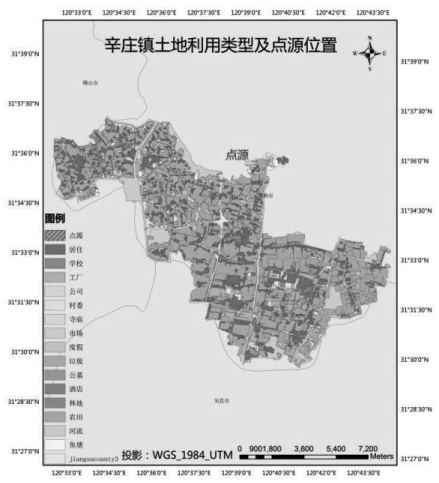

图 1 辛庄镇土地利用类型及垃圾焚烧厂位置

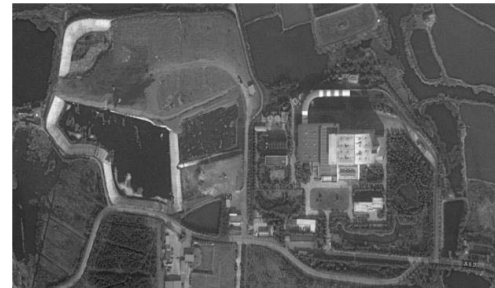

图 2 垃圾焚烧厂遥感影像

\section{2 基础数据}

基础数据分为三类: 排放源数据、气象数据、自然环 境数据, 清单及来源见表 1 。

\begin{tabular}{cc}
\multicolumn{2}{c}{ 表 1 数据清单及来源 } \\
\hline 数据 & 来源 \\
\hline 气象数据 & 中国气象科学数据服务网 \\
排放源数据 & 实地调研及文献[12-14] \\
自然环境数据 & 电子高程数据库 DEM、地表 \\
& 覆盖类型 LULC \\
\hline
\end{tabular}

经实地调研和参考现有文献中垃圾焚烧厂排放 源的典型值[12-14], 确定垃圾厂的排放源参数 (表 2), 烟图高度高于 $50 \mathrm{~m}$, 可以将排放源看成高架点源。

\begin{tabular}{ccc}
\multicolumn{3}{c}{ 表 2 排放源参数 } \\
\hline 参数 & 数值 & 单位 \\
\hline 通风量 & 60000 & $\mathrm{~m} / \mathrm{h}$ \\
烟囱排放颗粒物浓度 & 30 & $\mathrm{mg} / \mathrm{m}^{3}$ \\
烟图高度 & 60 & $\mathrm{~m}$ \\
烟图直径 & 1 & $\mathrm{~m}$ \\
排风风速 & 10.7 & $\mathrm{~m} / \mathrm{s}$ \\
排风温度 & 403 & $\mathrm{~K}$ \\
垃圾焚烧量 & 600 & $\mathrm{t} / \mathrm{d}$ \\
产灰率 & 0.04 & - \\
正常飞灰粒径 & $10-50$ & $\mu \mathrm{m}$
\end{tabular}

污染源的坐标采用 WGS 1984 UTM Zone 51N 系统显示, $(X, Y, Z)$ 为 $(278.295,3497.655,5)$, 颗粒物 和四种重金属排放量见表 3 。

\begin{tabular}{|c|c|c|}
\hline \multirow{3}{*}{ 坐标信息(m) } & $\mathrm{X}$ & 278.295 \\
\hline & $\mathrm{Y}$ & 3497.655 \\
\hline & Z & 5 \\
\hline \multirow{5}{*}{$\begin{array}{l}\text { 排放情况 } \\
\left(\mu \mathrm{g} / \mathbf{m}^{3}\right)\end{array}$} & PM & 138.89 \\
\hline & $\mathrm{Cu}$ & 0.1474 \\
\hline & $\mathrm{Cr}$ & 0.0502 \\
\hline & $\mathrm{Cd}$ & 0.0573 \\
\hline & $\mathrm{Ni}$ & 0.0257 \\
\hline
\end{tabular}




\section{Risk Analysis and Crisis Response in Big Data Era (RAC-16)}

2013 年江苏省常熟市辛庄镇的逐时地面气象数 据和高空气象数据来源为中国气象科学数据共享服 务网, 数据内容主要是风向、风速、温度、湿度、气 压、总云量、分云量、降水等。由于模拟的是辛庄镇 的气象状况，采用最靠近辛庄镇的 58343 (常州) 气 象站点的逐时气象数据作为地面气象数据; 采用 58238 (南京) 高空站作为高空气象数据。

自然环境数据包括地面数据和土地利用数据, 分 别从电子高程数据库和遥感影像中获取。按照实际利 用情况, 将辛庄镇土地利用类型划分成 15 类(图 1), 包括居住区、学校、工厂、垃圾厂、林地、农田、河 流、鱼塘等, 主要土地利用类型为农田、居住区。

\section{3 研究方法}

\subsubsection{CALPUFF 模拟方法}

CALPUFF 模型包括 CALMET 气象处理、CALPUFF 扩散处理和 CALPOST 后处理三个模块。CALMET 的原理是使用质量守恒方程诊断风场, 并输出逐时的 风场和温度场。CALMET 能处理各种复杂地形, 如 地形运动学影响调整、倾斜流调整、阻塞效应和闭合 效应调整等。在此模块中, CALMET 还对输入的风 场数据进行反距离加权、风廓线插值、两轮平滑和垂 直风速等操作, 并对温度和降水进行内插操作, 完善 数据, 输出参数。

CALPUFF 基本原理是高斯烟团模式，通过对时 间积分的方法来节约大量的计算时间 [15]。烟羽可以 看作是烟团的叠加, 计算原理是 “快照”, 假定瞬时 烟团不变, 输出各个计算点的污染浓度。烟团分裂采 用采样函数来描述烟团的空间轨迹、浓度分布; 烟云 抬升采用 Briggs 抬升公式, 考虑浮力和动量抬升[15]。 在每个时间点, 计算各个烟团对每个接收点浓度的贡 献, 其和即为此时刻接收点浓度。任何一个烟团对于 任何一个接收点的贡献, 均可以按照 3D 高斯模型计 算:

$$
C=\frac{Q}{2 \pi \sigma_{x} \sigma_{y}} g \exp \left[\frac{-d_{a}^{2}}{2 \sigma_{x}^{2}}\right] \exp \left[\frac{-d_{c}^{2}}{2 \sigma_{y}^{2}}\right]
$$

其中, $\mathrm{C}$ 是地面污染物浓度, $\mathrm{g} / \mathrm{m}^{3} ; \mathrm{Q}$ 是烟团中污染 物的质量, $\mathrm{g} ; \sigma_{\mathrm{x}}$ 是污染物在水平风向上高斯分布的 标准差, $m ; \sigma_{\mathrm{y}}$ 是污染物在垂直风向上高斯分布的标 准差, $m ; d_{a}$ 是水平方向上烟团中心到接收点的距离, $\mathrm{m} ; \mathrm{d}_{\mathrm{c}}$ 是垂直方向上烟团中心到接收点的距离, $\mathrm{m} ; \mathrm{g}$ 是垂向分布积分函数, $\mathrm{m}$ 。

CALPOST 后处理器主要是输出 CALPUFF 的处 理结果, 输出最后的浓度场以及沉降通量。

本论文在使用 CALMET 气象处理模块前, 将研
究区域划分成规则网格, 截取包括辛庄镇在内的 $18 \mathrm{~km} \times 18 \mathrm{~km}$ 的研究区域, 划分成边长为 $0.6 \mathrm{~km}$ 的 30 $\times 30$ 个正方形网格, 投影为 UTM 坐标系统。模拟场 景为: 垃圾焚烧厂每日处理垃圾 600 吨, 24 小时连 续运转排放废气。春、夏、秋、冬各选择一周进行模 拟。将 CALMET 所需的气象数据和土地利用数据输 入模型中对风场进行诊断, 输出大气稳定度、混合层 高度、逐时的风场等的三维风场和微气象场数据。然 后叠加排放源数据, 输入 CALPUFF 进行数值模拟, 最后通过 CALPOST 输出结果, 制图分析。

\subsection{2 健康风险计算方法}

为了评价该垃圾焚烧厂产生的大气重金属对居民的 健康风险, 采用美国环保局 (EPA) 推荐的人体暴露 健康风险评价模型[16]。设定重金属经呼吸途径进入 人体。将人群分为成年男性、成年女性和儿童三类, 根据类别选取参数。该模型的计算公式为:

$$
\begin{gathered}
A D D=(c \times I R \times E F \times E D) /(B W \times A T) \\
L A D D=(c \times I R \times E F \times E D) /(B W \times L T) \\
I L C R=L A D D \times S F \\
H Q=A D D / R f D
\end{gathered}
$$

其中 $A D D$ (average daily dose) 是非致癌物质的日 均暴露剂量, $\mathrm{mg} /(\mathrm{kg} \cdot \mathrm{d}$ ); $L A D D$ (lifetime average daily dose）是致癌物质的终身日均暴露剂量, $\mathrm{mg} /(\mathrm{kg} \cdot \mathrm{d})$; $\mathrm{c}$ 是污染物浓度, $\mathrm{mg} / \mathrm{m}^{3} ; I R$ 是呼吸速率, $\mathrm{m}^{3} / \mathrm{d} ; E F$ 是暴露频率 $\mathrm{d} / \mathrm{a} ; E D$ 是持续时间, $\mathrm{a} ; B W$ 是体重, $\mathrm{kg} ; A T$ 是平均暴露时间, $\mathrm{d} ; L T$ 是寿命, $\mathrm{d} ; I L C R$ 是致癌风险; $S F$ 是致癌斜率因子, $[\mathrm{mg} /(\mathrm{kg} \cdot \mathrm{d})]^{-1} ; H Q$ 是危害商值; $R f D$ 是参考剂量, $\mathrm{mg} /(\mathrm{kg} \cdot \mathrm{d})$ 。

ILCR 在 $10^{-6}-10^{-4}$ 之间时, 表示该重金属致癌风 险在可接受范围内; 大于该范围时, 为不可接受风险 水平。 $\mathrm{HQ} \leqslant 1$ 时, 该种重金属不存在非致癌健康风 险, $\mathrm{HQ}>1$ 时, 该重金属有非致癌风险。[5,17,18]

\section{3. 结果与讨论}

\section{1 重金属时空分布}

从空间分布来看, 除了浓度大小不同，四种重金 属浓度分布相似, 越靠近排放点源, 镉浓度越大, 随 着远离点源, 重金属浓度降低。四种重金属的平均浓 度大小顺序为铜 $>$ 镉 $>$ 铬>镍。其中铜浓度远大于其他 三种重金属, 镉和铬浓度较为相近, 镍最小。铜较之 镉、铬、镍在生活中使用更加普遍, 因此浓度最高。 以镉为例, 探讨大气中重金属浓度分布的季节差 异。如图 3 所示, 不同季节气候不同, 烟团扩散的方 
Risk Analysis and Crisis Response in Big Data Era (RAC-16)

向和轨迹不同, 空间分布差异明显。

春季镉浓度最大可达 $0.000088 \mu \mathrm{g} / \mathrm{m}^{3}$, 受风向影 响, 烟团主要向垃圾焚烧厂的西边和西北边扩散迁移, 在点源的西部镉浓度最高。夏季镉浓度最大可达 $0.000105 \mu \mathrm{g} / \mathrm{m}^{3}$, 比春季浓度更高。受风向影响, 烟 团主要向西北、东南、西南、东北四个方向扩散, 但 主要向西北和东南向迁移, 在垃圾焚烧厂的西北部镉 浓度最高。秋季镉浓度最大可达 $0.000034 \mu \mathrm{g} / \mathrm{m}^{3}$, 比 春季浓度更低。受风向影响, 烟团主要向东北方向扩 散, 其次是东南方向和西北方向, 少数向西南方向迁 移。在垃圾焚烧厂的东北部镉浓度最高。冬季镉浓度 最大可达 $0.000052 \mu \mathrm{g} / \mathrm{m}^{3}$, 只比秋季浓度稍高。受风 向影响, 烟团向西南、东南、西北三个方向扩散, 但 主要向东南向迁移, 在垃圾焚烧厂的东部镉浓度最高, 其次是西部。

其他重金属的空间分布与季节差异与镉类似, 篇 幅关系，不再赘述。

从时间分布来说, 垃圾焚烧厂排放的大气重金属 镉浓度大小为夏季 $>$ 春季 $>$ 冬季 $>$ 秋季, 春夏季节大气 镉浓度较高。一般来说, 由于夏季气温高, 污染物易 扩散稀释, 重金属浓度夏季一般低于冬季, 但不同重 金属在不同的区域有不同的表现特征[5]，不代表辛 庄镇重金属浓度分布情况不合常理。

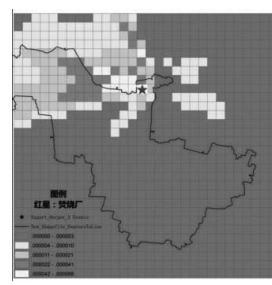

春

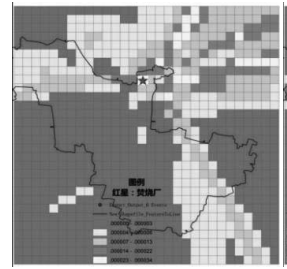

秋

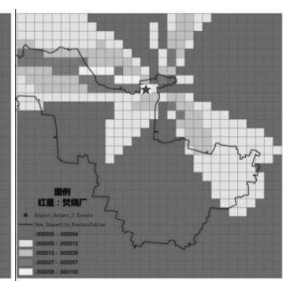

夏

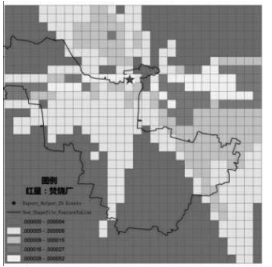

冬
图 3 镉浓度空间分布

\section{2 居民健康风险}

参考现有文献[19-21], 选取适合中国人群的暴露参数 如表 4, 通过美国综合风险数据库 (IRIS) 等获得致
癌斜率因子 $\mathrm{SF}$ 和参考剂量 RfD [16,21-24], 如表 5, 结合四种重金属的平均浓度值, 由公式(2)-(5)计算得 到三类人群的致癌及非致癌健康风险 (图 4, 图 5)。 表 4 呼吸途径暴露参数 [18-21]

\begin{tabular}{|c|c|c|c|c|}
\hline 项目 & $\begin{array}{l}\text { 成年 } \\
\text { 男性 }\end{array}$ & $\begin{array}{l}\text { 成年 } \\
\text { 女性 }\end{array}$ & \multicolumn{2}{|c|}{$\begin{array}{c}\text { 儿童 } \\
\text { (1-6) 岁 }\end{array}$} \\
\hline $\mathrm{IR} / \mathrm{m}^{3} \cdot \mathrm{d}^{-1}$ & 19.02 & 14.17 & \multicolumn{2}{|c|}{5} \\
\hline $\mathrm{BW} / \mathrm{kg}$ & 62.7 & 54.4 & \multicolumn{2}{|c|}{15} \\
\hline $\mathrm{EF} / \mathrm{d} \cdot \mathrm{a}^{-1}$ & 350 & 350 & \multicolumn{2}{|c|}{350} \\
\hline $\mathrm{ED} / \mathrm{a}$ & 30 & 30 & \multicolumn{2}{|c|}{6} \\
\hline LT（致癌）/d & $70 \times 365$ & $70 \times 365$ & \multicolumn{2}{|c|}{$70 \times 365$} \\
\hline AT (非致癌) /d & $30 \times 365$ & $30 \times 365$ & \multicolumn{2}{|c|}{$6 \times 365$} \\
\hline \multicolumn{5}{|c|}{ 表 5 四种重金属呼吸途径剂量-反应参数 [16,21-24] } \\
\hline \multicolumn{2}{|c|}{ 致癌物质 } & $\mathbf{C d}$ & $\mathrm{Cr}$ & $\mathbf{N i}$ \\
\hline \multicolumn{2}{|c|}{$\mathrm{SF} /\left[\mathrm{mg} \cdot(\mathrm{kg} \cdot \mathrm{d})^{-1}\right]^{-1}$} & 8.40 & 42 & 0.84 \\
\hline \multicolumn{2}{|c|}{ 非致癌物质 } & $\mathrm{Cu}$ & & \\
\hline \multicolumn{2}{|c|}{$\mathrm{RfD} / \mathrm{mg} \cdot(\mathrm{kg} \cdot \mathrm{d})^{-1}$} & $4.00 \times 10^{-2}$ & & \\
\hline
\end{tabular}

三种可致癌重金属镉, 铬, 镍的 ILCR 值都没有 超过 $10^{-4}$, 不存在致癌风险, 相对而言, 铬的致癌风 险较高。非致癌重金属铜的 HQ 值小于 1 , 风险可以 忽略。即辛庄镇垃圾焚烧厂排放的大气重金属对当地 居民没有造成大的健康风险。但是该研究基于短期的 浓度模拟, 长期的暴露风险还有待验证。在四种重金 属中应当重点关注铬的健康风险。

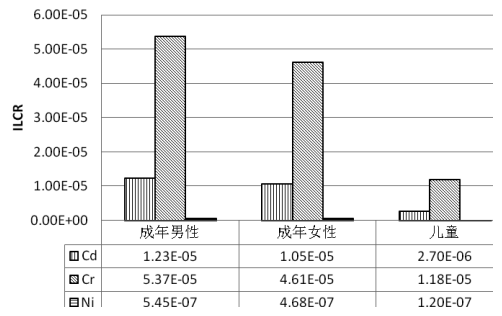

图 4 人群致癌健康风险

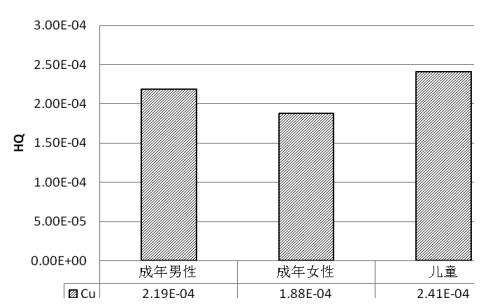

图 5 人群非致癌健康风险 
Risk Analysis and Crisis Response in Big Data Era (RAC-16)

\section{3 讨论}

大气重金属浓度大小表现为夏季 $>$ 春季 $>$ 冬季 $>$ 秋季, 而不是一般情况下夏季易扩散, 浓度最小, 可能与垃 圾焚烧厂实际焚烧的垃圾种类及当地的气候条件有 关，风速小烟团扩散迁移缓慢。四种重金属浓度空间 分布相似, 大小顺序为铜>镉>铬>镍, 这与当地垃圾 的种类和数量相关。垃圾焚烧厂在排放废气前进行了 无害化处理和除尘工序, 因此健康风险评价结果为镉, 铬, 镍的致癌风险在可接受范围; 非致癌重金属铜的 健康风险可以忽略, 即辛庄镇垃圾焚烧厂排放的大气 重金属对人体没有造成大的健康风险。但是本文只考 虑了呼吸暴露途径, 未考虑重金属通过干/湿沉降进 入土壤、地表水, 进而通过经口摄入及皮肤接触进入 人体的健康风险。因此, 计算结果可能是对实际风险 值的过小评价。

本研究使用 CALPUFF 模型模拟了辛庄镇垃圾 焚烧厂排放废气中重金属的迁移扩散, 分析了不同季 节大气重金属的浓度空间分布, 评价了大气重金属的 潜在健康风险。具有一定的参考价值, 并且为以后扩 大研究区域, 完善排放源种类和数量, 增加气象站数 量与精度提供经验。后续研究还需进行实地采样, 通 过模拟值与实际监测值的对比进行模型验证。

\section{4. 结论}

由四种重金属浓度的时空分布特征和健康风险分析, 得出以下结论:

（1）越靠近排放点源，大气重金属浓度越大, 远离点源, 重金属浓度降低。

（2）四种重金属的大气平均浓度大小顺序为铜> 镉>铬>镍。

（3）不同季节气候不同，烟团扩散的方向和轨 迹不同。垃圾焚烧厂的东北(秋)、东(冬)、西北(夏)、 西部（春）大气镉重金属浓度较高。

(4)不同季节大气镉浓度大小顺序为夏季>春季> 冬季 $>$ 秋季。

（5）不同重金属由于相同季节主导风向相同, 随着烟团扩散的方向和轨迹相同, 浓度分布相似。

（6）三种可致癌重金属镉, 铬, 镍致癌风险在 可接受范围; 非致癌重金属铜的健康风险可以忽略。 即辛庄镇垃圾焚烧厂排放的大气重金属对人体没有 造成大的健康风险。

\section{Acknowledgements}

This work was supported by the Key Technologies R\&D Program for the 12th Five-Year Plan (No. 2012BAJ24B04) of the Ministry of Science and Technology of People's Republic of China.

\section{致谢}

本研究得到了国家科技部 “十二五” 科技支撑课题 (No. 2012BAJ24B04)的资助。

\section{5.参考文献}

[1] 孔祥宇.成都市中心城区大气重金属污染特征分 析与区域扩散预测.成都理工大学, 2010.

[2] Cui Huiling, Yao Rentai, Xu Xiangjun. A tracer experiment study to evaluate the CALPUFF real time application in a near-field complex terrain setting. Atmospheric Environment, 2011, 45 (39): $7525-7532$.

[3] Joaquim Rovira, Montse Mari, Marta Schuhmacher. Environmental Pollution and Human Health Risks near a Hazardous Waste Landfill.Temporal Trends. Journal of Risk Analysis and Crisis Response, 2012, 2(1): 13-20.

[4] 宋宇, 陈家宜, 蔡旭晖. 石景山工业区 PM10 污 染对北京市影响的模拟计算.环境科学, 23:65 68,2002.

[5] 董婷, 李天昕, 赵秀阁, 等.某焦化厂周边大气 PM10 重金属来源及健康风险评价. 环境科学, 2014,35 (4): 1238-1244.

[6] Haofei Yu , AmyL. Stuart. Spatiotemporal distributions of ambient oxides of nitrogen, with implications for exposure inequality and urban design. Journal of the Air \& Waste Management Association, 2013, 63 (8):943-955.

[7] Hezhong Tian, Peipei Qiu. Current status and future trends of $\mathrm{SO}_{2}$ and $\mathrm{NO}_{x}$ pollution during the 12th FYP period in Guiyang city of China. Atmospheric Environment, 2013, 69:273-280.

[8] Ying Zhou, Jonathan I Levy, James K Hammitt. Estimating population exposure to power plant emissions using CALPUFF: a case study in Beijing, China. Atmospheric Environment, 2002, 37 (6):815-826.

[9] Ghannam K, EI-Fadel M. A framework for emissions source apportionment in industrial areas: MM5/CALPUFF in a near-field application Journal of the Air \& Waste Management Association, 2013, 63 (2):190-204.

[10] http://www.dfzb.suzhou.gov.cn/zsbl/1144927.htm

[11] http://www.dfzb.suzhou.gov.cn/zsbl/1145027.htm

[12] 王宗爽, 武婷, 段小丽, 等..环境科学研究, 2009, 22 (10): 1171-1175.

[13] 廖志恒, 孙家仁, 吴兑, 等. 基于不确定性分析 
Risk Analysis and Crisis Response in Big Data Era (RAC-16)

的垃圾焚烧烟气中重金属的土壤沉积及生态风 险评估. 环境科学, 2014,35 (6): 2264-2271.

[14] GB50051-2002 烟图设计规范.

[15] 国家环境保护环境影响评价数值模拟重点实验 室.CALPUFF 用户手册: 1-4.

[16] EPA. Risk assessment guidance for superfund volume I:human health evaluation manual. Supplemental guidance. "standard default exposure fators" interim final.EPA, 1991.

[17] USEPA. Risk Assessment Guidance for Superfund, vol. I: Human Health Evaluation Manual. EPA/540/1-89/002. Office of Solid Waste and Emergency Response, 1989.

[18] Ma J H, Singhirunnusorn W. Distribution and health risk assessment of heavy metals in surface dusts of Maha Sarakham Municipality. Procedia-Social and Behavioral Sciences, 2012,50: 280-293.

[19] 北京市环境保护科学研究院.DB11/T656-2009 场 地环境评价导则.北京: 北京市质量技术监督局, 2009.

[20] 王宗爽, 段小丽, 刘平, 等.环境健康风险评价 中我国居民暴露参数探讨. 环境科学研究, 2009,22 (10): 1164-1170.

[21] 杜金花, 张宜升, 何凌燕, 等.深圳某地区大气 PM2.5 中重金属的污染特征及健康风险评价. 环 境与健康杂志, 2012,29 (1): 77-79.

[22] USEPA. Integrated Risk Information System (IRIS) http://www.epa.gov/iris/.

[23] 陈灿灿, 卢新卫, 王利军, 等.宝鸡市街道灰尘 重金属污染的健康风险评价. 城市环境与城市生 态, 2011,24 (2): 35-38.

[24] 伯全金, 丁峰, 徐鹤, 等. 大气扩散 CALPUFF 模 型技术综述. 环境监测管理与技术, 2009, 21(3): 9-13、47. 